\title{
Does reason for referral to an ultra-high risk clinic predict transition to psychosis?
}

\author{
Simon Rice ${ }^{\text {a,b,c } \#}$ Andrea Polari ${ }^{\text {a,b,d,\# }}$ Andrew Thompson ${ }^{\text {e }}$, Jessica Hartmann ${ }^{\text {a,b }}$, \\ Patrick McGorry ${ }^{\text {a,b,d }} \&$ Barnaby Nelson ${ }^{\text {a,b,d* }}$
}

\section{Brief Report}

${ }^{a}$ Orygen, The National Centre of Excellence in Youth Mental Health

${ }^{\mathrm{b}}$ Centre for Youth Mental Health, The University of Melbourne, Australia

${ }^{\mathrm{c}}$ Youth Mood Clinic, Orygen Youth Health, Northwestern Mental Health, Melbourne

${ }^{\mathrm{d}}$ Personal Assessment and Crisis Evaluation (PACE) clinic, Orygen Youth Health, Northwestern Mental Health, Melbourne

${ }^{\mathrm{e}}$ Division of Mental Health and Wellbeing, The University of Warwick, Coventry, United Kingdom

${ }^{\text {\# }}$ Shared first author

This is the author manuscript accepted for publication and has undergone full peer review but has not been through the copyediting, typesetting, pagination and proofreading process, which may lead to differences between this version and the Version of Record. Please cite this article as doi: 10.1111/eip.12679

This article is protected by copyright. All rights reserved. 
*Corresponding Author: A/Prof Barnaby Nelson, Orygen, The National Centre of Excellence in Youth Mental Health, Centre for Youth Mental Health, The University of Melbourne, Locked Bag 10, Parkville, VIC 3052, AUSTRALIA. T: +03 9342 2800, E: barnaby.nelson.@orygen.org.au

\begin{abstract}
Aim: To examine reasons for referral to a specialist ultra-high risk for psychosis clinic and whether these reasons are associated with risk for subsequent transition to psychosis.

Methods: Data for 127 patients referred to the PACE clinic were collected by medical record audit. Time to transition to psychosis was calculated from date of referral to time at which the young person was judged by their treating team to be experiencing onset of first episode psychosis.
\end{abstract}

Results: Patients were primarily referred due to attenuated psychotic symptoms and depression (40.2\%). There was an association with transition, with those in the attenuated psychotic-symptom-only category being more likely to transition.

Conclusion: As well as attenuated psychotic symptoms, depression and anxiety were also important reasons for referral indicating that there is a broad clinical phenotype of young people presenting to UHR clinics. Clinical reason for referral may index level of risk for subsequent transition to psychosis.

Keywords: Ultra-high risk, psychosis, screening, youth mental health 


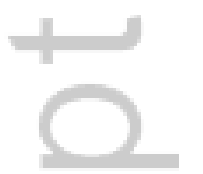

4 
Understanding the risk phenotype of psychosis has been a key goal of research examining the emergence of psychotic disorder (van Os \& Tamminga, 2007). A focus on the prodromal phase of illness has sought to identify cases deemed to be at ultra-high risk (UHR) for psychosis, with the goal of providing intervention to prevent subsequent transition to a psychotic disorder, and to investigate aetiological mechanisms driving the onset of psychotic disorder (Nelson et al., 2013). Efficient early detection and intervention requires valid identification of at-risk individuals who may have a poor clinical prognosis (Ziermans, Schothorst, Sprong, \& van Engeland, 2011). Identifying predictors of different outcome trajectories is an ongoing challenge for research in the UHR field (Yung \& Lin, 2016).

Population-based studies suggest that psychotic symptoms are associated with a range of diagnoses other than those within the schizophrenia spectrum (Johns et al., 2004; Varghese et al., 2009). Indeed, long-term follow-up indicates that many UHR patients who do not transition to psychosis continue to experience attenuated psychotic symptoms or develop other psychiatric diagnoses (Lin et al., 2015). It has been speculated that cases in whom attenuated psychotic symptoms are thought to be secondary to emerging or existing mood or anxiety disorders, so-called 'incidental' attenuated psychotic symptoms (Yung et al., 2006), may be at lower risk of developing a diagnosable psychotic disorder (vans Os \& Linscott, 2012; Yung et al., 2006) and that this may contribute to the observed lower rate of transition to psychosis in more recent UHR cohorts (Hartmann et al., 2016; Nelson et al., 2013; Yung et al., 2006). In two general population studies, attenuated psychotic symptoms have been found to be a risk factor for severe psychopathology generally, not just psychotic disorder, characterised by high comorbidity and suicidal behaviour (Kelleher et al., 2012a; 2012b). 
Recent research has also focused on 'pre-test' characteristics of UHR patients (e.g., demographic characteristics, socioeconomic status, referral source, etc.) as indexing risk for subsequent psychosis (Fusar-Poli et al., 2016; Fusar-Poli 2017; O'Donoghue et al., 2015; Wiltink, Velthorst, Nelson, McGorry, \& Yung, 2015). Fusar Poli and colleagues (2016) reported that referral source is a key pre-test variable in identifying UHR patients most at risk of psychosis, with referrals received from outside mental health services (i.e., selfreferral, caregivers or relatives, school) associated with lower transition risk than referrals from mental health services. What remains unclear, and yet to be researched, is whether reason for referral to UHR clinics relates to transition outcome. The aim of the present study was to examine reasons for referral to a specialist UHR clinic, and determine whether reason for referral was associated with subsequent transition to psychosis. Given those with incidental 'psychotic symptoms' (and therefore at lower transition risk, as described above) are generally referred to UHR clinics due to non-psychotic symptoms plus attenuated psychotic symptoms, we predicted a lower transition rate for this group relative to those with attenuated psychotic symptoms alone.

\section{Method}

\section{Participants}

Study participants were 127 patients referred to the PACE clinic, Orygen Youth Health, Melbourne, Australia (Yung et al., 2007), between 2003 and 2007. Referrals were either self-referrals or from a variety of external sources, including general practitioners, school welfare coordinators, schools, private psychiatrists, families/friends, and other mental health services. All patients were UHR for psychosis, meeting at least one of the three UHR 
groups: Vulnerability Group (Family history of psychosis, Schizotypal Disorder), Attenuated Psychotic Symptoms (APS), Brief Limited Intermittent Psychotic Symptoms (BLIPS) as operationalised using the CAARMS (Yung et al., 2005). All had experienced a decline in functioning (i.e., a 30\% drop in SOFAS in the year prior to referral), or had chronic low functioning (less than 50 on the SOFAS for at least 1 month in the year prior to referral). The time to follow-up ranged from 1.2 to 6.5 years with a median of 4.5 years.

\section{Data source}

A file audit was undertaken (see O'Connor et al., 2016 for full details). A consecutive series of patients who subsequently transitioned to a psychotic disorder between 2003 and 2008 were identified (60 cases). A randomly selected sample from the same time period who did not transition to a psychotic disorder were also included (67 controls). The reason for referral was recorded using a specifically designed file audit tool.

\section{Data analysis}

Reason for referral categories were developed (see Table 1) for major symptom domains. A symptom severity index was calculated by multiplying each of the four CAARMS severity subscale scores (i.e., disturbed speech, non-bizarre ideation, perceptual abnormality, unusual thought content) by the symptom frequency score, and summing these four values. Chi-square analysis examined an association between reason for referral and transition to psychosis for the 'APS only', 'APS + other symptoms', and 'other symptoms' groups and the relative risk of transition was calculated. Kaplan-Meier analysis was undertaken to examine differences in time to transition comparing those with APS only $(n=42)$ and those with APS with other symptoms $(n=71)$. 


\section{Results}

Mean age at referral was 18.20 years $(S D=8.42)$. There was no difference in age according to those who subsequently transitioned to psychosis. The sample included marginally fewer males $(41.7 \%, \mathrm{n}=53)$. Most $(90.6 \%, \mathrm{n}=115)$ of the sample had never been married and most were living with parents $(68.5 \%, n=87)$. Most patients $(72.4 \%)$ met the APS UHR intake group, with $8.7 \%$ meeting the Vulnerability group criteria and only 1 $(0.8 \%)$ patient meeting BLIPS criteria (see Figure 1). There was no mean difference in duration of symptoms prior to assessment between the group APS only ( $M=37.92, S D=40.62$ weeks $)$ and APS + other symptoms $(M=37.94, S D=47.06$ weeks $)$, nor was there any categorical association between referral source and the APS only and APS + other symptoms groups. Data on severity of attenuated psychotic symptoms were available for 50 cases (39.5\%). Of these cases, there was no difference in symptom severity between those who transitioned and did not transition, however those in the APS only group reported a higher total symptom score $(M=38.70 . S D=27.13)$ compared to those in the APS + other symptoms group $(M=27.13, S D=12.48), F(1,46)=7.12, p=.011$.

\section{Insert figure 1 here}

When the specific symptomology constituting the reason for referral to PACE was explored, patients referred due to 'APS only' (33.1\%) or 'APS + depression' $(40.2 \%)$ comprised almost three quarters of referrals (see Table 1). When three overarching referral categories (i.e., 'APS only', 'APS + other symptoms', and 'other symptoms') were examined by transition status (yes/no), a trend-level association was observed $C^{2}(1, N=127)=5.54$, $p=.063$. Transition rates for the three groups were $61.9 \%, 40.8 \%$ and $35.7 \%$ respectively for 
the 'APS only' (total n=42), 'APS + other symptoms' (total n=71), and 'other symptoms' (total $n=14$ ) groups.

\section{Insert Table 1 here}

When the 14 cases with a 'non-APS' referral reason were excluded, Kaplan-Meier analysis indicated a significant effect of referral group on time to transition (log rank $\left.C^{2}(1)=4.70, p=0.031\right)$ - see Figures 2 and 3 . Those in the 'APS only' category were proportionally more likely to transition earlier (relative risk $=1.52,95 \% \mathrm{CI}=1.05-2.19$; estimated $M=32.72$ months, $S D=5.14$ ) relative to those in the 'APS + other' category (relative risk $=0.66,95 \% \mathrm{CI}=0.46-0.95$; estimated $M=50.12$ months, $S D=4.29)$.

\section{Insert Figures 2 and 3 here}

\section{Discussion}

The main reason for referral to the specialist UHR clinic was a combination of attenuated psychotic symptoms and depressive symptoms. Consistent with our hypothesis, those referred due to attenuated psychotic symptoms + other symptoms were at lower risk of transitioning to psychosis than those referred due to attenuated psychotic symptoms alone.

The findings show that patients are referred to UHR clinics for a variety of reasons, not only attenuated psychotic symptoms. It also seems that the clinical reason for referral may index level of risk for subsequent transition to psychosis, with those referred purely due to concern about attenuated psychotic symptoms being at higher risk than those referred due to attenuated psychotic symptoms in combination with an array of other symptoms. One might speculate that the latter group are more representative of clinical cases in whom 
attenuated psychotic symptoms are secondary to emerging or existing mood or anxiety disorders (vans Os \& Linscott, 2012), sometimes referred to as 'incidental' attenuated psychotic symptoms (Yung et al., 2006), and are therefore at lower risk of developing an independent and diagnosable psychotic disorder. For example, the aetiology of some clinical presentations for those identified as UHR positive by the CAARMS (i.e., persecutory ideas, non-bizarre ideation, ideas of guilt) may be largely explained by pervasive anxious rumination, persistent low mood (i.e., major depression), or exposure to psychological trauma (i.e., complex trauma) rather than symptoms indicative of an emerging psychotic disorder. Our interpretation assumes that for the group with APS + other symptoms, the attenuated psychotic symptoms are of secondary clinical significance. This may not necessarily be the case. Unfortunately the reason for referral variable in the present dataset did not differentiate primary and secondary referral reasons. In order to advance the field, we recommend that future studies examine this.

In terms of clinical implications, identifying the reason for referral may be another form of 'pre-test risk enrichment'. In this way the present results build on the findings reported in Fusar-Poli et al. (2016) who found that ethnicity and source of referral were strong pre-test risk enrichment variables. Further, it is possible that patients with APS + other symptoms may be more responsive to treatment, as shown in lower transition risk. It may be that current forms of psychotherapy may be more effective in targeting non-psychotic symptoms (i.e., mood/anxiety), which then has a positive flow on effect to reducing transition risk.

The present analyses are limited by the large variability in group size (reason for referral). Therefore, larger groupings were formed based on the most frequent reasons for 
referral, reducing this variability, and replication in a larger cohort is needed. It is also possible that severity of attenuated psychotic symptoms may have played a role in transition risk and confounded the effect of reason for referral. However, we were not able to examine this possibility given the lack of available data (only available on $39 \%$ of the sample.) This issue should be considered in future research into this topic. In summary, findings of this study support the view that pre-test characteristics in this clinical population play an important role in indexing level of psychosis risk. Additional focussed research is indicated.

\section{References}

Fusar-Poli, P., Rutigliano, G., Stahl, D., Schmidt, A., Ramella-Cravaro, V., Hitesh, S., \& McGuire, P. (2016). Deconstructing pretest risk enrichment to optimize prediction of psychosis in individuals at clinical high risk. JAMA Psychiatry, 73(12), 1260-1267.

Fusar-Poli, P. (2017). The clinical high-risk state for psychosis (CHR-P), version II. Schizophrenia Bulletin, 43, 44-47.

Guloksuz, S., van Nierop, M., Lieb, R., van Winkel, R., Wittchen, H. U., \& van Os, J. (2015). Evidence that the presence of psychosis in non-psychotic disorder is environmentdependent and mediated by severity of non-psychotic psychopathology. Psychological Medicine, 45(11), 2389-2401.

Johns, L. C., Cannon, M., Singleton, N., Murray, R. M., Farrell, M., Brugha, T., ... \& Meltzer, H. (2004). Prevalence and correlates of self-reported psychotic symptoms in the British population. The British Journal of Psychiatry, 185(4), 298-305.

Kelleher, I., Lynch, F., Harley, M., Molloy, C., Roddy, S., Fitzpatrick, C., \& Cannon, M. (2012a). Psychotic symptoms in adolescence index risk for suicidal behavior: 
findings from 2 population-based case-control clinical interview studies. Archives of General Psychiatry, 69(12), 1277-1283.

Kelleher, I., Keeley, H., Corcoran, P., Lynch, F., Fitzpatrick, C., Devlin, N., ... \& Arseneault, L. (2012b). Clinicopathological significance of psychotic experiences in nonpsychotic young people: evidence from four population-based studies. The British Journal of Psychiatry, 201(1), 26-32.

Lin, A., Wood, S. J., Nelson, B., Beavan, A., McGorry, P., \& Yung, A. R. (2015). Outcomes of nontransitioned cases in a sample at ultra-high risk for psychosis. American Journal of Psychiatry, 172(3), 249-258.

Hartmann, J. A., Yuen, H. P., McGorry, P. D., Yung, A. R., Lin, A., Wood, S. J., ... \& Nelson, B. (2016). Declining transition rates to psychotic disorder in "ultra-high risk" clients: investigation of a dilution effect. Schizophrenia Research, 170(1), 130136.

Nelson, B., Yuen, H. P., Wood, S. J., Lin, A., Spiliotacopoulos, D., Bruxner, A., ... \& Francey, S. M. (2013). Long-term follow-up of a group at ultra high risk ("prodromal") for psychosis: the PACE 400 study. JAMA Psychiatry, 70(8), 793802.

Morosini, P. L., Magliano, L., Brambilla, L., Ugolini, S., \& Pioli, R. (2000). Development, reliability and acceptability of a new version of the DSM-IV Social and Occupational Functioning Assessment Scale (SOFAS) to assess routine social funtioning. Acta Psychiatrica Scandinavica, 101(4), 323-329.

This article is protected by copyright. All rights reserved. 
O'Connor, K., Nelson, B., Lin, A., Wood, S. J., Yung, A., \& Thompson, A. (2016). Are UHR patients who present with hallucinations alone at lower risk of transition to psychosis? Psychiatry Research, 235, 177-196.

O'Donoghue, B., Nelson, B., Yuen, H. P., Lane, A., Wood, S., Thompson, A., ... \& Yung, A. R. (2015). Social environmental risk factors for transition to psychosis in an ultrahigh risk population. Schizophrenia Research, 161(2), 150-155.

Rekhi, G., Rapisarda, A., \& Lee, J. (2017). Impact of distress related to attenuated psychotic symptoms in individuals at ultra-high risk of psychosis: Findings from the Longitudinal Youth at Risk Study. Early Intervention in Psychiatry. Early view, doi: 10.1111/eip.1245.1

Rössler, W., Hengartner, M. P., Ajdacic-Gross, V., Haker, H., Gamma, A., \& Angst, J. (2011). Sub-clinical psychosis symptoms in young adults are risk factors for subsequent common mental disorders. Schizophrenia Research, 131(1), 18-23.

van Os, J., \& Tamminga, C. (2007). Deconstructing psychosis. Schizophrenia Bulletin, 33(4), 861-862.

van Os, J., \& Linscott, R. J. (2012). Introduction: the extended psychosis phenotyperelationship with schizophrenia and with ultrahigh risk status for psychosis. Schizophrenia Bulletin, 38(2), 227-230.

Varghese, D., Scott, J., Welham, J., Bor, W., Najman, J., O'callaghan, M., ... \& McGrath, J. (2009). Psychotic-like experiences in major depression and anxiety disorders: a population-based survey in young adults. Schizophrenia Bulletin, 37(2), 389-393.

Wiltink, S., Velthorst, E., Nelson, B., McGorry, P. M., \& Yung, A. R. (2015). Declining transition rates to psychosis: the contribution of potential changes in referral 
pathways to an ultra-high-risk service. Early Intervention in Psychiatry, 9(3), 200206.

Yung, A., Yuen, H., McGorry P., et al. (2005). Mapping the onset of psychosis: the Comprehensive Assessment of At-Risk Mental States. Australia and New Zealand Journal Psychiatry. 39, 964-971.

Yung, A. R., Stanford, C., Cosgrave, E., Killackey, E., Phillips, L., Nelson, B., \& McGorry, P. D. (2006). Testing the ultra-high risk (prodromal) criteria for the prediction of psychosis in a clinical sample of young people. Schizophrenia Research, 84(1), 5766.

Yung, A. R., McGorry, P. D., Francey, S. M., Nelson, B., Baker, K., Phillips, L. J., ... \& Amminger, G. P. (2007). PACE: a specialised service for young people at risk of psychotic disorders. Medical Journal of Australia, 187(7), S43-S46.

Yung, A. R., Nelson, B., Thompson, A., \& Wood, S. J. (2010). The psychosis threshold in Ultra High Risk (prodromal) research: is it valid? Schizophrenia Research, 120, 1-6.

Yung, A. R., Yuen, H. P., Berger, G., Francey, S., Hung, T. C., Nelson, B., ... \& McGorry, P. (2007). Declining transition rate in ultra-high risk (prodromal) services: dilution or reduction of risk? Schizophrenia Bulletin, 33(3), 673-681.

Yung, A. R., \& Lin, A. (2016). Psychotic experiences and their significance. World Psychiatry, 15(2), 130-131.

Ziermans, T. B., Schothorst, P. F., Sprong, M., \& van Engeland, H. (2011). Transition and remission in adolescents at ultra-high risk for psychosis. Schizophrenia Research, 126(1), 58-64.

This article is protected by copyright. All rights reserved. 


\section{University Library}

\section{- M M N E R VA A gateway to Melbourne's research publications}

Minerva Access is the Institutional Repository of The University of Melbourne

Author/s:

Rice, S;Polari, A;Thompson, A;Hartmann, J;McGorry, P;Nelson, B

Title:

Does reason for referral to an ultra-high risk clinic predict transition to psychosis?

Date:

2019-04-01

Citation:

Rice, S., Polari, A., Thompson, A., Hartmann, J., McGorry, P. \& Nelson, B. (2019). Does reason for referral to an ultra-high risk clinic predict transition to psychosis?. EARLY

INTERVENTION IN PSYCHIATRY, 13 (2), pp.318-321. https://doi.org/10.1111/eip.12679.

Persistent Link:

http://hdl.handle.net/11343/284036 